\title{
Switching from concomitant therapy to tafluprost/timolol fixed combination
}

This article was published in the following Dove Press journal: Clinical Ophthalmology

\section{Kenji Inoue \\ Taku Kawashima \\ Seiki Katakura \\ Kyoko Shidara \\ Takayuki Fujimoto \\ Hiromi Kohmoto \\ Minako Shiokawa \\ Ryo Moriyama \\ Ryoko Okayama}

Inouye Eye Hospital, Tokyo, Japan
Correspondence: Kenji Inoue Inouye Eye Hospital, 4-3 Kanda-Surugadai, Chiyoda-ku Tokyo I0I-0062, Japan

Tel +8I3 329509 I।

Fax +81332950917

Email inoue-k@inouye-eye.or.jp
Purpose: To evaluate the efficacy and safety of tafluprost/timolol fixed combination (TTFC). Study design: A prospective clinical study.

Methods: Twenty-eight patients (28 eyes) with primary open-angle glaucoma, who had used tafluprost and timolol gel for at least 3 months with good adherence, were enrolled. Concomitant administration of tafluprost and timolol was switched to TTFC without a washout period. The intraocular pressure (IOP), blood pressure, pulse rate, and ocular signs were compared between before switching (baseline), and 4 and 8 weeks after switching. A questionnaire survey was also performed 4 weeks after switching to investigate ocular comfort and patient preferences.

Results: The IOP showed no significant change after switching to TTFC $(14.8 \pm 2.8,14.6 \pm 3.4$, and $14.8 \pm 3.7 \mathrm{mmHg}$ at baseline, Week 4 , and Week 8 , respectively). The pulse rate and systolic blood pressure showed no changes, but diastolic blood pressure was significantly lower at Week 8. At baseline, fluorescein staining revealed corneal abnormalities in 3 patients, which resolved by Week 8 in 1 patient. Hyperemia was noted in 2 patients at baseline, and this also resolved by Week 8 in 1 patient. Three patients discontinued study treatment for the following reasons ( 1 patient each): blurred vision; ocular irritation, eyelid erythema, and asthenopia; and loss to follow-up from Week 8 . The questionnaire survey revealed no significant differences between the 2 treatments, although more patients preferred TTFC.

Conclusion: Among 28 patients enrolled, only 2 patients discontinued the study treatment due to adverse reactions. In patients whose adherence was considered relatively good to concomitant therapy, switching to TTFC achieved similar IOP control with good safety and a high level of patient acceptance.

Keywords: tafluprost/timolol fixed combination, switching, intraocular pressure, adverse reactions

\section{Introduction}

Glaucoma is a progressive disease which requires lifelong treatment to maintain visual function. A primary treatment for primary open-angle glaucoma is topical medication to reduce intraocular pressure (IOP). It is commonly recommended to initiate the treatment with monotherapy in glaucoma guidelines, and sometimes the addition of a second drug should be considered when the initial monotherapy has not succeeded in reaching the target IOP. ${ }^{1}$ However, multiple topical medications may reduce adherence and increase unfavorable side effects. Thus, the merits of fixed combination medications include improved adherence, compared with concomitant administration of individual medications, associated with a decrease of dosing and fewer adverse reactions.

Tafluprost/timolol fixed combination (TTFC) eye drops contain both tafluprost and timolol in a single topical preparation, which achieves favorable control of IOP 
with a good safety profile. ${ }^{2-4}$ The preserved formulation of TTFC has been available in Japan since 2014. The preserved TTFC was developed to optimize $\mathrm{pH}$ and improve the ocular penetration of timolol, in order to compensate for possible attenuation of the effect of timolol associated with a lower dosing frequency. ${ }^{5}$ Clinical trials performed in Japan have confirmed non-inferiority of this fixed combination to concomitant therapy. ${ }^{2}$ However, comparison of TTFC with concomitant therapy in the real-world Japanese clinical setting has not been performed.

Thus, the present prospective study was initiated to compare safety and reduction of the IOP with TTFC therapy versus concomitant administration of tafluprost and timolol in the routine clinical setting. Patients with primary open-angle glaucoma were enrolled and switched from concomitant administration to TTFC. Only patients with good adherence to concomitant therapy were enrolled in the study, which may reduce the influence of improvement in adherence to medication after switching. In addition, we evaluated ocular comfort and patient preferences for the 2 regimens by performing a questionnaire survey.

\section{Subjects and methods}

We obtained written informed consent from 34 patients with primary open-angle glaucoma (including normal tension glaucoma) who had shown good adherence (instilling $\geq 75 \%$ of the prescribed dosage, assessed by self-report) to concomitant treatment with tafluprost and timolol gel for at least 3 months. Subjects were enrolled at Inouye Eye Hospital (Ochanomizu, Tokyo, Japan) between February 2015 and October 2016. Inclusion criteria: 1) age $\geq 20$ years at the time of giving informed consent; 2) male or female; 3) patients who have used tafluprost and timolol gel concomitantly for at least 3 months before the study; 4) other glaucoma ophthalmic solutions (carbonic anhydrase inhibitor, $\alpha_{1}$-blocker, or $\alpha_{2}$-agonist) were allowed, but the patient must have been on the same regimen for more than 3 months before the study. Main exclusion criteria: 1) patients who have any corneal abnormalities or diseases that may interfere with determination of IOP by applanation tomometry; 2) patients who have a history of keratorefractive surgery; 3 ) patients with active extraocular disease or inflammation/infection of the eye/eyelid; 4) patients with a history of glaucoma surgery (eg, selective laser trabeculoplasty, filtering surgery, or trabeculotomy); 5) patients undergoing anterior or posterior ocular surgery within 3 months before the study; 6) patients scheduled for use of prohibited concomitant drugs or therapies during the study. If both eyes satisfied these criteria, the eye with the higher IOP before switching (baseline) was assessed, while the right eye was assessed if the IOP was the same in both eyes. Among the 34 patients giving written informed consent, 6 patients did not satisfy all of the inclusion criteria or met exclusion criteria and were excluded from the study, leaving 28 patients.

Concomitant administration of benzalkonium chloride (BAK) containing tafluprost $0.0015 \%$ (Tapros $^{\circledR}$; Santen Pharmaceutical Co. Ltd, Osaka, Japan) (once daily at night) and timolol $0.5 \%$ gel-forming solution (Timoptol ${ }^{\circledR} \mathrm{XE}$; Santen Pharmaceutical Co. Ltd) (once daily in the morning) was discontinued, and the BAK containing tafluprost $0.0015 \% /$ timolol $0.5 \%$ fixed combination $\left(\right.$ Tapcom $^{\circledR}$; Santen Pharmaceutical Co. Ltd) was started once daily in the morning without a washout period. Patients were allowed to continue any other eye drops that were being used to treat glaucoma 3 months before baseline. IOP was determined with a Goldmann applanation tonometer before switching and at Weeks 4 and 8 after switching, while the blood pressure and pulse rate were measured by an automated sphygmomanometer (Udex super Type, Elquest Corporation, Chiba, Japan) at the same times. The measurement time for each patient was decided based on the time of baseline measurement, and the measurements were performed at the same time, as far as possible. The patients were classified into 3 groups according to the change of IOP from baseline to Week 4 or 8 after switching: 1) decrease of $\geq 2 \mathrm{mmHg}$; 2) change of within $2 \mathrm{mmHg}$; and 3) increase of $\geq 2 \mathrm{mmHg}$. In addition, a questionnaire survey was performed at Week 4 after switching to investigate the ocular comfort and patients' preference about the fixed combination and their previous medications. The questionnaires: 1-1) "Did you ever forget your medication during the 1 week prior to switching to TTFC? (Yes/No)"; 1-2) if the patients answered "yes" to Question 1-1, "Has the frequency of missed doses decreased after switching to TTFC? (Decreased/No difference/Increased)"; 2) "Do you notice any difference in the following ocular symptoms after switching medication? (Less/Same as before/More)" for irritation, itching, blurred vision, and hyperemia; 3-1) "Do you prefer the eye drops before switching or the eye drops after switching? (Before switching/Either is fine/ After switching)"; 3-2) "Give the reason for your answer to Question 3-1 (No irritation/No itching/No blurred vision/No hyperemia/Lower dosing frequency/Other reasons)". Corneal findings (fluorescein staining), conjunctival hyperemia, adverse reactions, and reasons for discontinuing the study treatment were analyzed at Weeks 0,4 , and 8 . Corneal sign changes were rated according to area-density grades ${ }^{6}$ and 
conjunctival hyperemia was evaluated by comparing the treated eye with reference images. ${ }^{7}$

For analysis of IOP, the Last Observation Carried Forward (LOCF) method was used for discontinued patients. Repeated-measures analysis of variance (ANOVA) models were used to evaluate the time-wise changes from baseline in IOP. The paired $t$-test was used to compare blood pressure, and pulse rate between baseline and Week 4 or 8 after switching. Probability values of less than 0.05 were considered significant. The sample size was based on the following assumptions with reference to previous studies: ${ }^{2}$ the change of IOP was predicted to be $0 \mathrm{mmHg}$ after switching, the noninferiority margin was set as $1.5 \mathrm{mmHg}$, standard deviation was $2.5 \mathrm{mmHg}$, and power was $80 \%$ (paired $t$-test with a $5 \%$ level of significance). Calculations based on these assumptions revealed that 24 patients were required for the study, so a target of at least 30 patients was set by taking possible dropouts into consideration. The study protocol was approved by the Institutional Review Board of Inouye Eye Hospital and conducted in accordance with the Declaration of Helsinki and Ethical Guidelines for Medical and Health Research Involving Human Subjects of the Japanese Ministry of Health, Labour and Welfare. Candidate subjects were given an explanation of the details of the study, and provided written informed consent before enrollment. This study was registered with the UMIN clinical trials registry (ID: UMIN 000016052).

\section{Results}

The 28 patients enrolled in the study comprised 12 men and 16 women aged $71.3 \pm 10.6$ years (mean $\pm \mathrm{SD}$; range: 47 to 87 years), including 20 patients with primary open-angle glaucoma and 8 patients with normal tension glaucoma. The baseline IOP of the 28 patients was $14.8 \pm 2.8 \mathrm{mmHg}$ (range: 10 to $21 \mathrm{mmHg}$ ). They were using a mean of $2.6 \pm 0.7$ ophthalmic medications at baseline, with 14,10 , and 4 patients using 2 (only tafluprost + timolol gel), 3 (other than these 2 drugs, 4: brinzolamide, 3: dorzolamide, 2: brimonidine, and 1: bunazosin), and 4 drugs (2: brinzolamide + bunazosin, 2: brinzolamide + brimonidine), respectively.

The IOP was determined in 26 patients at Weeks 4 and 8 , and the LOCF method was used in IOP at Week 8 . The IOP was $14.6 \pm 3.4 \mathrm{mmHg}$ and $14.8 \pm 3.7 \mathrm{mmHg}$ at Weeks 4 and 8 after switching, respectively, showing no significant difference from the baseline pressure ( $p=0.421$, ANOVA) (Figure 1). At Week 4, a decrease of the IOP by $\geq 2 \mathrm{mmHg}$, a change within $2 \mathrm{mmHg}$, or an increase by $\geq 2 \mathrm{mmHg}$ was observed in 4 patients (15.4\%), 20 patients $(76.9 \%)$, and 2 patients (7.7\%), respectively (Figure 2). At Week 8 , a decrease of the IOP by $\geq 2 \mathrm{mmHg}$, change within $2 \mathrm{mmHg}$, and increase by $\geq 2 \mathrm{mmHg}$ was observed in 5 patients $(19.2 \%)$, 17 patients $(65.4 \%)$, and 4 patients $(15.4 \%)$, respectively (Figure 2).

The pulse rate and systolic blood pressure showed no significant difference between baseline and Weeks 4 and 8 after switching medication ( $p=0.949$ and $p=0.443, p=0.905$ and $p=0.275$, respectively) (Table 1 ). Diastolic blood pressure decreased significantly from $72 \pm 10 \mathrm{mmHg}$ at baseline to $67 \pm 10 \mathrm{mmHg}$ at Week 8 after switching $(p<0.05)$, without showing a significant difference at Week $4(p=0.461)$.

Corneal abnormalities (area grade 1, density grade 1 ) were noted in 3 patients by fluorescein staining at baseline. At Week 8 after switching medication, the abnormality had resolved in 1 patient, but persisted in 2 patients (area grade 1 and density grade 1). Conjunctival hyperemia (score of 1) was observed in 2 patients at baseline. It resolved in 1 patient by Week 8 and persisted in 1 patient (score of 1).

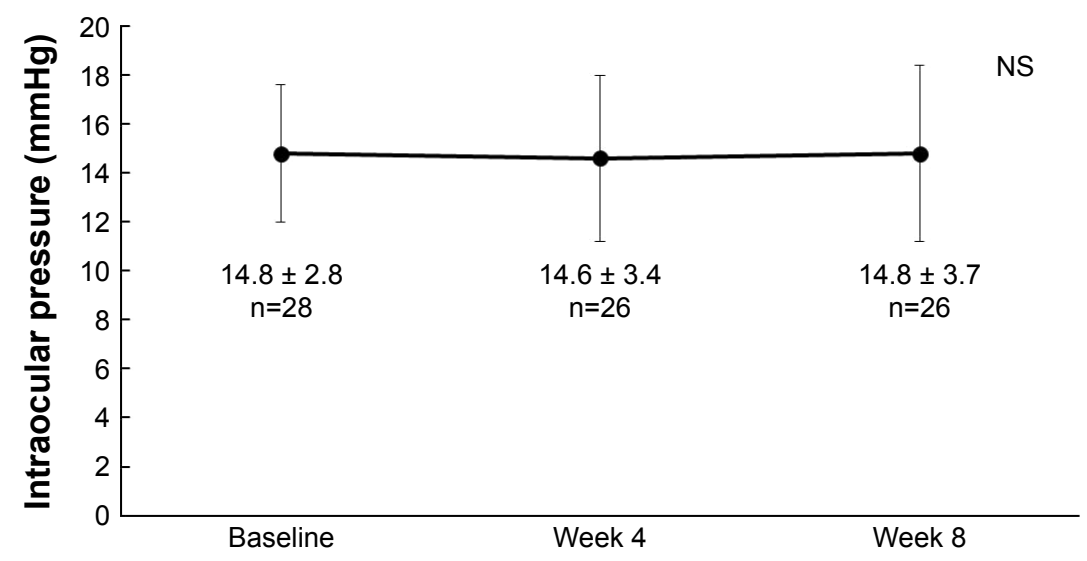

Figure I Changes of mean intraocular pressure before and after switching to the tafluprost/timolol fixed combination.

Note: Data represent the mean \pm standard deviation.

Abbreviation: NS, not significant. 


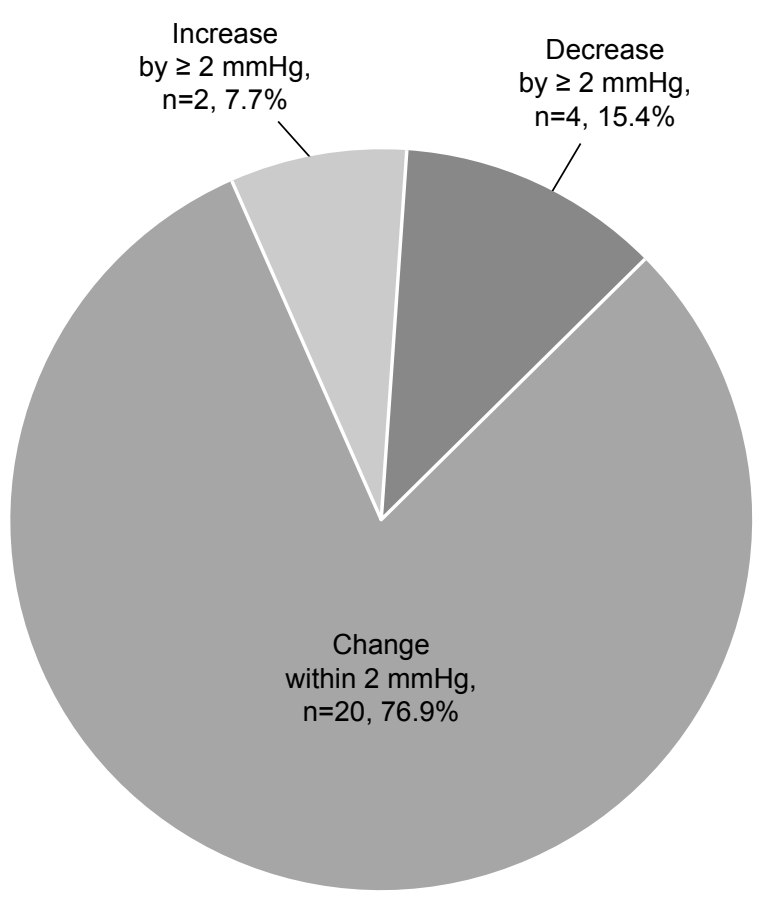

Week $4(n=26)$

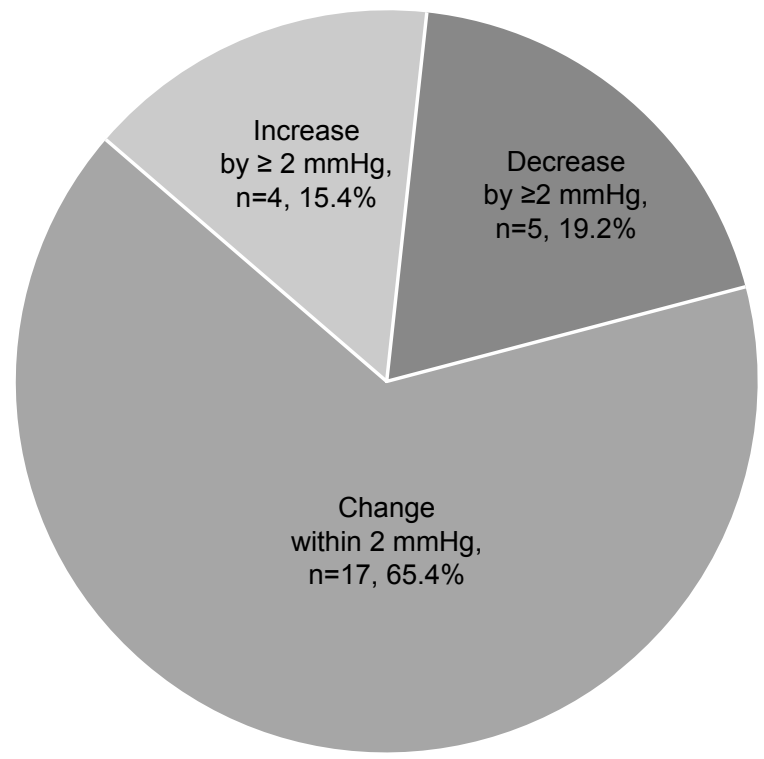

Week $8(n=26)$

Figure 2 Frequency of intraocular pressure alterations after switching to the tafluprost/timolol fixed combination.

The questionnaire survey was not completed by 2 patients who discontinued the study treatment before or at Week 4 due to adverse reactions. Among 26 patients, 1 did not return the answers for Week 4, so 25 patients were included in the analysis of questionnaire data. Twenty of 25 patients (80.0\%) answered they had not forgotten their medication during the 1 week prior to TTFC initiation, and 5 patients $(20.0 \%)$ answered they had. Among these 5 patients, 3 patients (60.0\%) answered the frequency of missing dose decreased after switching to TTFC, and 2 patients $(40.0 \%)$ answered "No difference". To questions regarding the differences of ocular symptoms (irritation, itching, blurred vision, and hyperemia), most patients answered that there was no difference ("Same as before", Table 2). For the preference for unfixed combination ("before switching") or fixed combination ("after switching"), 2 patients $(8.0 \%), 17$ patients $(68.0 \%)$, and 6 patients

Table I Blood pressure and pulse rate before and after switching to the tafluprost/timolol fixed combination

\begin{tabular}{|c|c|c|c|c|c|}
\hline & Baseline & Week 4 & $\begin{array}{l}\text { P-value } \\
\text { (baseline vs } \\
\text { Week 4) }\end{array}$ & Week 8 & 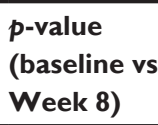 \\
\hline \multicolumn{6}{|c|}{ Blood pressure $(\mathrm{mmHg})$} \\
\hline Systolic & $127 \pm 17$ & $127 \pm 21$ & 0.905 & $122 \pm 23$ & 0.275 \\
\hline Diastolic & $72 \pm 10$ & $70 \pm 11$ & 0.461 & $67 \pm 10$ & $<0.05$ \\
\hline $\begin{array}{l}\text { Pulse rate } \\
\text { (min) }\end{array}$ & $70 \pm 11$ & $69 \pm 13$ & 0.949 & $73 \pm 13$ & 0.443 \\
\hline
\end{tabular}

Notes: Data presented as mean \pm SD.
(24.0\%) answered unfixed combination, fixed combination, and with no preference, respectively. Among the 2 patients who preferred the unfixed medications, 1 patient answered that the reason was "no blurred vision" and neither of them selected the other options ("no irritation", "no itching", "no hyperemia", and "lower dosing frequency"). Among the 6 patients who answered that either regimen was fine, 2 patients selected "lower dosing frequency" and none of the 6 patients selected the other options. Among the 17 patients who preferred the fixed combination, 16 patients selected "lower dosing frequency" as the reason, while 3 patients answered "no irritation of the eye" and 2 patients answered "no blurred vision". Other answers included "it is less sticky".

Adverse reactions were observed in 2 of the 34 patients who received TTFC treatment. One patient experienced ocular irritation, eyelid erythema, and asthenopia before Week 4 and discontinued treatment, while 1 patient discontinued treatment due to blurred vision at Week 4 . The adverse reactions of these 2 patients resolved after stopping the study drug.

Table 2 Results of the questionnaire survey

\begin{tabular}{lrrrr}
\hline & Irritation & Itching & $\begin{array}{l}\text { Blurred } \\
\text { vision }\end{array}$ & Hyperemia \\
\hline Improved & 5 & $\mathrm{I}$ & 6 & 2 \\
Same as before & 15 & $2 \mathrm{I}$ & 17 & $2 \mathrm{I}$ \\
Worse & 4 & 2 & $\mathrm{I}$ & $\mathrm{I}$ \\
No answer & $\mathrm{I}$ & $\mathrm{I}$ & $\mathrm{I}$ & । \\
\hline
\end{tabular}


In addition, 1 patient did not return for assessment at Week 8 and was lost to follow-up. Therefore, a total of 3 patients discontinued treatment before the end of the study.

\section{Discussion}

We investigated the efficacy and safety of TTFC in a realworld setting. The patients in this study were Japanese patients with glaucoma who responded with good adherence to concomitant use of the individual preparations before enrollment in the present study. As a result, we found that there was no significant change in the IOP after switching to TTFC from concomitant use of two separate preparations and no serious adverse reactions occurred after switching. In addition, the patients preferred TTFC to concomitant use of 2 preparations.

There were various previous reports regarding the effects on the IOP of prostaglandin/timolol fixed combinations compared to unfixed combinations. ${ }^{8-13}$ These clinical studies varied in study design, such as switching studies or parallel studies, and also showed slightly different results, ie, some studies reported that the fixed combinations were more effective than unfixed combinations, and others not. To the best of our knowledge, there was no clinical report in a real-world setting evaluating the effect on IOP of TTFC compared to unfixed combinations of its individual active ingredients. The current study showed that switching to TTFC achieved similar IOP control. This result might be consistent with previous reports that the IOP-lowering efficacy of TTFC is non-inferior to its unfixed combination in the Phase III clinical trials. ${ }^{2,4}$

Prostaglandin/timolol fixed combinations usually have clinical equivalence to unfixed combinations, although the results were slightly different than those described above..$^{8-13}$ Theoretically, in a once-daily fixed combination, the daily dose of timolol is halved compared to the standard twicedaily timolol preparation, which might cause the attenuation of efficacy. On the other hand, fixed combinations are favorable for adherence which may practically compensate for the attenuation of efficacy. This may explain the mixed results of previous studies.

We enrolled patients, in the present study, whose adherence to medication was considered relatively good before switching, in order to reduce the potential influence of any change in adherence. Although recorded as patient self-reporting, only 3 of the 25 patients reported a decrease of missed doses after switching, suggesting that there was little influence of adherence on the results. Therefore, good intraocular distribution of timolol after instillation of $\mathrm{TTFC}^{5,14}$ may affect IOP control of TTFC positively.
In the present study, both systemic and ocular adverse reactions were evaluated to assess the safety profile. Compared to our previous studies of other prostaglandin/timolol fixed combinations, less adverse reactions were observed in this study (6.8\% in latanoprost/timolol, ${ }^{11} 16.3 \%$ in travoprost/ timolol, ${ }^{13} 5.9 \%$ in this TTFC study). The difference in the incidence of adverse reactions may be partly explained by the shorter observation period of the present study compared with the previous studies ( 8 weeks vs 12 months ${ }^{11}$ and 6 months ${ }^{13}$ ), and by use of multiple medications in the study on the travoprost/timolol fixed combination ${ }^{13}$ (the prostaglandin analog was either latanoprost, travoprost, tafluprost, or isopropyl unoprostone and the $\beta$-blocker was either timolol, carteolol, levobunolol, or nipradilol). In this study, we found that the pulse rate and systolic blood pressure did not change after switching, but the diastolic blood pressure decreased significantly. With regard to drug safety evaluation, a diastolic blood pressure $\geq 105 \mathrm{mmHg}$ or $\leq 50 \mathrm{mmHg}$ or a change of diastolic blood pressure from baseline by $\geq 15 \mathrm{mmHg}$ is clinically relevant. Therefore, although the reason for the decrease of diastolic pressure is unknown, it was not a clinical problem because the actual change was only $5 \mathrm{mmHg}$ and the diastolic pressure was $67 \pm 10 \mathrm{mmHg}$ at Week 8, and we concluded that this change was not a safety concern. These findings suggest the safety of TTFC is similar to that of other fixed combinations.

Fewer adverse reactions are a potential advantage of fixed combination medications over concomitant therapy. In our previous study of a latanoprost/timolol fixed combination, ${ }^{11}$ irritation of the eyes resolved in $54.3 \%$ of 236 of the patients, ${ }^{11}$ while hyperemia disappeared in $50 \%$ of the patients in our study of a travoprost/timolol fixed combination. ${ }^{13}$ In the present study, corneal abnormalities or hyperemia were only observed in a few patients at baseline, but resolved after switching to TTFC in $33.3 \%$ and $50.0 \%$ of the patients with such problems, respectively. This improvement of ocular symptoms may be attributable to reduced exposure to preservatives (eg, BAK) associated with the lower daily dosing frequency of TTFC.

When patients were asked about their preference for concomitant use of 2 preparations or fixed combination therapy, $68.0 \%, 82.1 \%$, and $54.8 \%$ preferred fixed combinations of tafluprost/timolol, latanoprost/timolol, ${ }^{11}$ and travoprost/timolol, ${ }^{13}$ respectively, and the reason for preferring each fixed combination was reduction of dosing frequency. In the present study, the patients had a favorable impression of TTFC, suggesting that both fewer adverse reactions and improved adherence can be expected with this medication. 
The main limitation of this study was a short observation period ( 8 weeks). An investigation with a longer observation period is required to confirm the present findings. The design was another limitation, since it was a switching study and not a head-to-head comparison, which means that bias favoring the medication after switching cannot be ruled out in the evaluation of efficacy and the questionnaire survey. Furthermore, we assessed the adherence by patient self-report, since there is no accurate method to measure true patient adherence. The IOP control might have been affected by the influence of the change in adherence during the study period.

In summary, we evaluated the efficacy and safety of TTFC for glaucoma in comparison with concomitant administration of the individual medications by switching from concomitant therapy to TTFC without a washout period. In these patients, TTFC maintained IOP at the same level as before the switch and was also well tolerated with good adherence, since only 2 patients discontinued it due to adverse reactions. Accordingly, switching from concomitant therapy with 2 preparations to a fixed combination might be clinically useful.

\section{Acknowledgments}

This was an investigator-industry collaborative study, funded by Santen Pharmaceutical Co., Ltd. The funding organization participated in the design of the study and in preparation and review of the manuscript. Collection of data and statistical analysis were conducted by Inouye Eye Hospital. The funding organization was not involved in these processes. The standard photographs of hyperemia were provided by Santen Pharmaceutical Co., Ltd.

\section{Disclosure}

K Inoue: lecture fees (Santen, Alcon Japan, Otsuka, Pfizer, Senju, Kowa), writing fees (Santen, Alcon Japan), and consulting fees (Santen, Alcon Japan, Pfizer, Senju, Kowa, Kaken, Nitten). T Kawashima: writing fee (Santen). M Shiokawa; lecture fee (Alcon Japan) and consulting fee (Otsuka). Okayama: lecture fee (Santen). The authors report no other conflicts of interest in this work.

Clinical Ophthalmology

\section{Publish your work in this journal}

Clinical Ophthalmology is an international, peer-reviewed journal covering all subspecialties within ophthalmology. Key topics include: Optometry; Visual science; Pharmacology and drug therapy in eye diseases; Basic Sciences; Primary and Secondary eye care; Patient Safety and Quality of Care Improvements. This journal is indexed on Submit your manuscript here: http://www.dovepress.com/clinical-ophthalmology-journal

\section{References}

1. [No authors listed]. European Glaucoma Society Terminology and Guidelines for Glaucoma, 4th Edition - Chapter 3: Treatment principles and options Supported by the EGS Foundation. $\mathrm{Br} J$ Ophthalmol. 2017;101(6):130-195.

2. Kuwayama Y, DE-111 Collaborative Trial Group. Phase III doublemasked study of fixed combination tafluprost $0.0015 \% /$ timolol $0.5 \%$ (DE-111) versus tafluprost $0.0015 \%$ alone or given concomitantly with timolol $0.5 \%$ in primary open angle glaucoma and ocular hypertension. Atarashii Ganka. 2013;30(8):1185-1194.

3. Kuwayama Y, DE-III Collaborative Trial Group. A long-term, openlabel study of fixed combination tafluprost $0.0015 \%$ /timolol $0.5 \%$ (DE-III) in patients with open-angle glaucoma or ocular hypertension. Atarashii Ganka. 2015;32(1):133-143.

4. Hollo G, Hommer A, Lopez AA, Ropo A. Efficacy, safety, and tolerability of preservative-free fixed combination of tafluprost $0.0015 \%$ / timolol $0.5 \%$ versus concomitant use of the ingredients. $J$ Ocul Pharmacol Ther. 2014;30(6):468-475.

5. Ueda K, Tonouchi A, Fukano Y, Asada H, Kawazu K. Tafluprost $0.0015 \% /$ timolol $0.5 \%$ combination ophthalmic solution (DE-111 ophthalmic solution) formulation design and intraocular penetration in rats. Atarashii Ganka. 2013;30(12):1761-1766.

6. Miyata K, Amano A, Sawa M, Nishida T. A novel grading method for superficial punctate keratopathy magnitude and its correlation with corneal epithelial permeability. Arch Ophthalmol. 2003;121(22):1537-1539.

7. Kanamoto T, Kiuchi Y, Tanito M, et al. Comparison of the toxicity profile of benzalkonium chloride-preserved tafluprost and SofZiapreserved travoprost applied to the ocular surface. J Ocul Pharmacol Ther. 2015;31(3):156-164.

8. Quaranta L, Biagioli E, Riva I, et al. Prostaglandin analogs and timololfixed versus unfixed combinations or monotherapy for open-angle glaucoma: a systematic review and meta-analysis. J Ocul Pharmacol Ther. 2013;29(4):382-389.

9. Webers CA, Beckers HJ, Zeegers MP, Nuijts RM, Hendrikse F, Schouten JS. The intraocular pressure-lowering effect of prostaglandin analogs combined with topical $\beta$-blocker therapy: a systematic review and meta-analysis. Ophthalmology. 2010;117(11):2067-2074.

10. Inoue K, Okayama R, Higa R, Sawada H, Wakamura M, Tomita G. Ocular hypotensive effects and safety over 3 months of switching from an unfixed combination to latanoprost $0.005 \%$ /timolol maleate $0.5 \%$ fixed combination. J Ocul Pharmacol Ther. 2011;27(6):581-587.

11. Inoue K, Okayama R, Higa R, Wakakura M, Tomita G. Assessment of ocular hypotensive effect and safety 12 months after changing from an unfixed combination to a latanoprost $0.005 \%$ + timolol maleate $0.5 \%$ fixed combination. Clin Ophthalmol. 2012;6:607-612.

12. Inoue K, Okayama R, Higa R, Tomita G. Efficacy and safety of switching to latanoprost $0.005 \%$-timolol maleate $0.5 \%$ fixed-combination eyedrops from an unfixed combination for 36 months. Clin Ophthalmol. 2014;8:1275-1279.

13. Inoue K, Setogawa A, Higa R, Moriyama R, Wakakura M, Tomita G. Ocular hypotensive effect and safety of travoprost $0.004 \% /$ timolol maleate $0.5 \%$ fixed combination after change of treatment regimen from $\beta$-blockers and prostaglandin analogs. Clin Ophthalmol. 2012;6:231-235.

14. Fuwa M, Ueda K, Akaishi T, et al. Advantages of efficacy and safety of fixed-dose tafluprost/timolol combination over fixed-dose latanoprsot/ timolol combination. PLoS One. 2016;11:e0158797.

\section{Dovepress}

PubMed Central and CAS, and is the official journal of The Society of Clinical Ophthalmology (SCO). The manuscript management system is completely online and includes a very quick and fair peer-review system, which is all easy to use. Visit http://www.dovepress.com/ testimonials.php to read real quotes from published authors. 